\title{
A New Optimization Algorithm for Speed of Electric Vehicle Based on Fuzzy PID Control
}

\author{
Jiheng Jiang \\ School of Electrical and Electronic Engineering, North China Electric Power University, Beijing \\ 102206, China \\ sdjiangjiheng@163.com
}

Keywords: Fuzzy theory, Mamdani inference system, Electric Vehicle, Internet of Vehicles

\begin{abstract}
Combined with the power characteristics of electric vehicles and the modern internet of vehicles, this paper proposes a new optimization algorithm for speed of electric vehicles based on fuzzy theory and the conventional PID control rules. We study characteristics of different factors in the process of driving, wherein the Park equation is used to establish the relationship between motor current and dynamic situation. The selection of input variables takes Internet of Vehicles technology into account. In consideration of the target of energy conservation, the acceleration is defined as the output variable of the whole optimization algorithm, which is proved to be accurate in all circumstances. The examination with Matlab FIS illustrates the new algorithm has a better control effect, providing a new method for the fuzzy PID control on the speed of electric motor.
\end{abstract}

\section{Introduction}

In recent years, the development of technology makes the function of car turns into Autonomous Intelligence, rather than the old Action Intelligence. In the prior research of autopilot on vehicles, speed control technology attracts scholars and engineers from various countries. Traditional speed control technology has been widely applied on their research. But with the advent of the era of Internet and the rapid spread of electric vehicles, numerous kinds of control technology in automotive engineering will constantly update. Considering the highly interconnected information and the particularity of dynamic system on the Electric Vehicle (EV), it is necessary for us to study the speed control technology of electric vehicle.

Speed control technology can be traced back to a part of pilot model, which is classic velocity model. American scholar R.Wade Allen put forward a model of speed control based on the relative velocity in $1997^{[1]}$. Researchers in Japan Nissan company established a model of speed control based on relative distance in $1998^{[2]}$. In these models, input variables are all the variables from the surrounding environment, which is collected by sensors. And the widely used speed control technology is also completely based on the following model of traffic operations, showing the severe blindness and limitations. In addition, driving is a complex nonlinear time-varying process, and the existing control methods mainly use the traditional PID method, of which three parameters cannot change according to the state of the system real time optimization. Applied on the speed of complex dynamic systems, this method will lead to severe overshoot or oscillation. On this basis, the application of fuzzy PID control for complex system control provides a new way. This paper will combine the dynamic performance of the electric car with the requirements of both safety and economy, introducing an optimization algorithm of the speed for fuzzy PID control process of electric motor.

\section{The Dynamic Model of Electric Vehicle}

The equation can be established by the following analysis. As for a vehicle moving on the road, the complicated relationship of force is,

$$
F_{t}=F_{f}+F_{w}+F_{i}+F_{j}
$$

where $F_{t}$ is driving force, $F_{f}$ is rolling resistance while moving, $F_{w}$ is air resistance, $F_{i}$ is ramp resistance while moving, $F_{j}$ is accelerating resistance while moving. 
In general control systems, the control phase can only manage the driving force and the acceleration of the vehicle while the other resistance remain uncontrollable. And for the accelerating resistance, it can be calculated in accordance with,

$$
F_{j}=\frac{\delta G}{g} \frac{d v}{d t}
$$

wherein $\delta$ is mass conversion coefficient, indicates the equivalent mass increase due to the energy consumption caused by the rotating parts of vehicles. The driving power of electric vehicle is produced by electric motor. At present, most of the electric vehicles in China are using permanent magnet synchronous motor. According to Parker transformation equation, we can get the voltage equation of the synchronous motor:

$$
\left\{\begin{array}{l}
u_{d}=R_{s} i_{d}+L_{d} \frac{\mathrm{d} i_{d}}{\mathrm{~d} t}-\omega_{r} L_{q} i_{q} \\
u_{q}=R_{s} i_{q}+L_{q} \frac{\mathrm{d} i_{q}}{\mathrm{~d} t}+\omega L_{d} i_{d}+\omega_{r} \psi_{f}
\end{array}\right.
$$

The torque equation of synchronous motor is:

$$
\left\{\begin{array}{l}
T_{e}=\frac{3}{2} n_{p}\left[\psi_{f} i_{q}+\left(L_{d}-L_{q}\right) i_{d} i_{q}\right] \\
T_{e}=-\frac{3}{2} n_{p}\left[\frac{u_{d} \psi_{f}}{\omega_{r} L_{d}}+\frac{u_{d} u_{q}\left(L_{d}-L_{q}\right)}{\omega_{r}^{2} L_{d} L_{q}}\right]
\end{array}\right.
$$

Wherein $u_{d}, u_{q}$ are motor terminal voltage of axis $\mathrm{d}$ and axis $\mathrm{q}, i_{d}, i_{q}$ are stator current of axis $\mathrm{d}$ and axis q, $L_{d}, L_{q}$ are inductance of axis d and axis q, $\psi_{f}$ is rotor permanent magnet flux linkage, $n_{p}$ is pole number of electric motor, $\omega_{r}$ is angular velocity of electric motor, $R_{s}$ is stator resistance.

The existing commonly used motor control technology is vector control. The torque speed is adjusted by the current of D, Q axis which is controlled by SVPWM module. The driving force of electric car is in direct proportion to the torque of the motor. Therefore, the acceleration of the vehicle can be changed by controlling the output current of the synchronous motor. Further, it is proved that the capability and life span of the battery are closely related to discharge rate. The higher the rate is, the faster the battery capability decays. Meanwhile, a high discharge rate causes aging and damage easily when the SOC of the battery is very low. Therefore, the properties of the power battery must be taken into consideration while optimizing. Here the algorithm of this paper will take the acceleration of the vehicle as the output controlled variable.

\section{The Optimization Algorithm}

\subsection{The Framework of the Optimization Algorithm}

The existing speed control technology of automatic driving is not a simple overlay of sensor circuit, central control system and cruise control system. The integration of the different functions of the three is bound to be a new trend. The dynamic part of a certain predetermined speed can be controlled by the system. Therefore, Speed optimization algorithm research has become a new hot spot. In order to illustrate the theory in detail, we divide a whole intelligent fuzzy PID module into two parts - speed optimization and motor control, which is showed in Figure 1.

Based on the analysis above, we found that the simple speed control focuses only on planning of the steady state while the difference in process volume was ignored, which goes against the management of energy and motor control. Therefore, we assume acceleration as the output reference of the whole algorithm, and then provide input for motor control.

Except for the economic efficiency, safety and the driving habits should be take into consideration.

With the development of internet of vehicles and the help of ITS and RFID technology, the interconnection between vehicles to internet (V2I), vehicles to human being (V2H) was increased. 

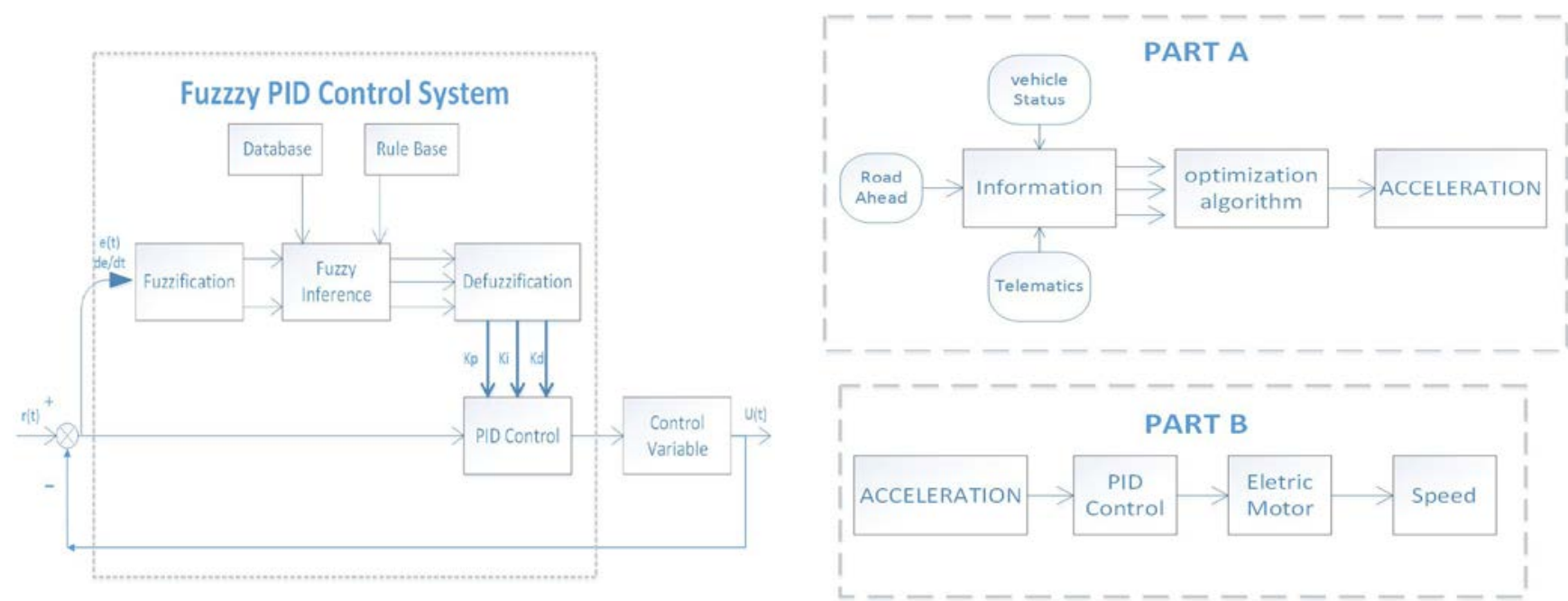

Fig.1 The Division of Fuzzy PID Control System

The driver can easily get information about the road condition and early warning in advance.

Based on the existing car-following model and dynamic equation, we herein put forward the framework of the optimization algorithm. The output variable is the acceleration $a_{c}$. The factors that should be considered are,

a) current speed $V_{c}(\mathrm{~m} / \mathrm{s})$

b) SOC of battery $S_{o c}(\%)$

c) discharge rate $I_{c}(\mathrm{C} / \mathrm{n})$

d) speed of the vehicle in front $V_{B}(\mathrm{~m})$, distance $D_{B}(\mathrm{~m} / \mathrm{s})$

e) traffic density $\rho_{V}(\mathrm{pcu} / \mathrm{km})$

f) distance to the change of road (ramp, turning, passive stop) $D_{S}(\mathrm{~m})$

Among the six factors, the first three belongs to vehicle itself, of which the information can be collected by monitoring unit. The speed of the vehicle in front and the distance can be detected by radar. The information of the last two factors can be obtained by internet vehicles.

\subsection{Application of Mamdani Fuzzy Inference System}

In the actual fuzzy PID control system, we input vector $r(t)$ and get three coefficient $K_{p}, T_{i}, T_{d}$ by calculating with fuzzy rules. We can in this way determine the control process and control the speed of the motor, which has good dynamic characteristics such as lack of steady-state error and fast response. Here, due to the similarity of the input variables and the solution principle, we focus on the solution of optimal acceleration instead of the concrete process of PID control module. Since driving is a complex dicision making process, the relationship between influencial factors and driving states is nonlinear, where Mamdani fuzzy inference system can be applied. This system is to achieve the inference from the input to the output by a set of rules. It helps to establish the identification system of the input data space with human intervention.

$$
\begin{gathered}
R^{i} \text { :if } x^{1} \text { is } A_{1}^{i} \text { and } x^{2} \text { is } A_{2}^{i} \cdots \text { and } x^{j} \text { is } A_{j}^{i} \\
\text { then } y^{i}=H^{i},
\end{gathered}
$$

where, $x^{j}$ is represents the inputed variable, $A_{j}^{i}$ is represents the subset of $x j, y^{i}$ is represents the corresponding local output of rule $\mathrm{i}, H^{i}$ is represents the fuzzy subset.

\subsection{The Processing of Input Variables.}

Six variables put forward by the above affect the driving strategy. But they are not the independent variables from each other. In order to reduce the number of input variables, this model merges some of them, generating new five variables:

a) The current speed $V_{c}$. 
b) The ratio between discharge Rate and battery $R_{\text {battery }}=I_{C} / S_{o c}$.

c) The ratio between difference of speed and distance $R_{V D}=\left(V_{C}-V_{B}\right) / D_{B}$.

d) Vehicle density $\rho_{V}$.

e) The probability of parking $P_{s t}=V_{C} / D_{S}$.

\subsection{The Determination of Membership Functions}

To simplify the process, only five variables are selected as the elements of the fuzzy set, based on the one-dimensional fuzzy inference system, which means the rate of change is not involved. To ensure the continuous stability of control performance, the membership function of input adopts the gaussian function of uniform superposition without gap. In order to improve the precision of parameter identification for the output, acceleration, the membership function of output adopts the triangle function of uniform superposition without gap.

Vehicle condition and the practical experience can be reference to determine the universe of all variables which can be set as $[-1,1]$, according to the mixture normalization. The following process are on the basis of normalization.

Due to the input is in a large quantity, the description of inputs is divided into three levels, that is $\{\mathrm{NB}$ (minus), Z(zero), $\mathrm{PB}$ (plus) $\}$. The description of outputs is $\{\mathrm{NB}$ (minus left), NS(minus), Z(zero), PS(plus), $\mathrm{PB}$ (plus right)\}.

\subsection{The Establishment of the Fuzzy Control Rules Table}

According to the driving experiences and the characteristics of the actual driving, Speed optimization rules are built (as simply explained below).

Table 1 Rule Base of the Mamdani Fuzzy Inference System

\begin{tabular}{cccccc}
\hline$V_{\mathrm{c}}$ & $R_{\text {battery }}$ & $R_{V D}$ & $\rho_{V}$ & $P_{\text {st }}$ & $a_{c}$ \\
\hline NB & NB & NB & NB & NB & PB \\
NB & NB & NB & NB & Z & PB \\
NB & PB & NB & Z & PB & PS \\
PB & PB & PB & PB & PB & NB \\
PB & PB & PB & Z & PB & NB \\
PB & PB & Z & PB & Z & NS \\
PB & NB & PB & Z & PB & PS \\
Z & Z & Z & Z & Z & Z \\
\hline
\end{tabular}

\section{The Simulation Research}

On the basis of the algorithm which are proposed before, we has realized the simulation of the algorithm in the FIS sysytem of MATLAB. With the simulation program adopting GUI tools, the surface of acceleration control is obtained under different parameter Settings. These results are shown in Figure 2.

Figures shows that, when the other three variables are set to zero, the controller maintains acceleration between 0 to 0.2 , very stable, where the two independent variables are minus. And the acceleration changes quickly in the middle transitional region, showing the high discernibility. The peak is very obvious in the part of the diagonal. So we can come to the conclusion that changes of acceleration are small and smooth when all the parameters are basically identical. 

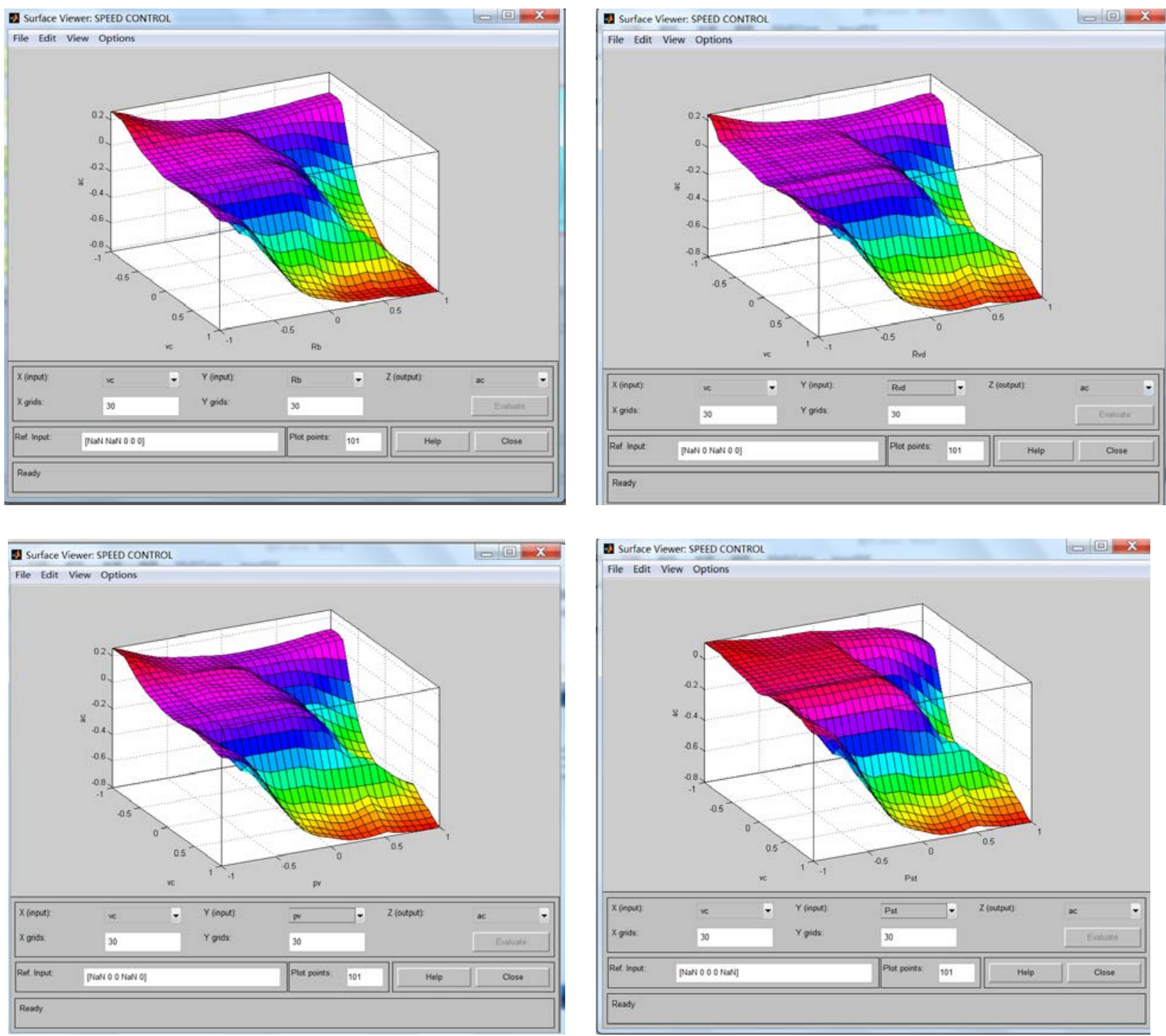

Fig.2 Results of Simulation

\section{Summary}

In this paper, the influence degree of the various inputs is fully combined with the speed optimization model for the target of controlling the acceleration. Controlling the speed indirectly has reached the requirement of safety and economy, which makes the overshoot lower, response faster. The simulation results of control surface conform to the actual experience, illustrating this set of algorithm has better practicability and accuracy. We can provide a referential input for the further motor PID control.

\section{References}

[1] Martens M H, Compte S, Kaptein Nico A. The Effects of Road Design on Speed Behaviour: a literature review[R], 1997.

[2] Rachel M.Malinauskas. The Intelligent Driver Model: Analysis and Application to adaptive cruise control[D]. 2014.05.

[3] YING H. Structure and Stability Analysis of General Mamdani Fuzzy Dynamic Models. International Journal of Intelligent Systems, 2005, NO.20: P. 103-125. 